\title{
Correction: Advances and potential pitfalls of oncolytic viruses expressing immunomodulatory transgene therapy for malignant gliomas
}

Qing Zhang and Fusheng Liu

Correction to: Cell Death and Disease

https://doi.org/10.1038/s41419-020-2696-5

published online 25 June 2020

Since publication the authors have noticed that there was an error in the author information. Fusheng Liu is the only corresponding author, while Qing Zhang is the first author.

This has been corrected in the PDF and HTML versions.

Published online: 23 November 2020 